Full Length Article

\title{
Tuning the optical response of cross-linked Fe@Au nanoparticles
}

\author{
Najme Ahmadi ${ }^{\mathrm{a}}$, Reza Poursalehi ${ }^{\mathrm{a}}$, Andrei Kirilyuk ${ }^{\mathrm{b}}$, Mohammad Kazem Moravvej-Farshi ${ }^{\mathrm{c}, *}$ \\ ${ }^{\text {a }}$ Nano Plasmo-Photonic Research Group, Department of Material Engineering, Tarbiat Modares University, Tehran 1411713116, Iran \\ ${ }^{\mathrm{b}}$ FELIX Laboratory, Radboud University, Toernooiveld 7c, 6525 ED Nijmegen, the Netherlands \\ ${ }^{\mathrm{c}}$ Nano Plasmo-Photonic Research Group, Faculty of Electrical and Computer Engineering, Tarbiat Modares University, Tehran 1411713116, Iran
}

\section{A R T I C L E I N F O}

\section{Keywords:}

Magnetic-plasmonics nanostructures

Arrayed Fe@Au nanoparticles

Magneto-optical effects

\begin{abstract}
A B S T R A C T
We are proposing a novel method to form thin films that are composed of ordered arrays of iron-gold core-shell (Fe@Au) nanoparticles with tunable optical responses. The method is based on the crosslinking of the adjacent Fe@Au nanoparticles using bifunctional group organic molecules. Molecules of m-phenylenediamine (MPD) as conjugated diamines can crosslink the adjacent Fe@Au nanoparticles. Experimental results show that the larger the Fe@Au/MPD mass ratio, the more intense the corresponding plasmon resonance. Images of the optical microscopy and FE-SEM show that there is a tradeoff between the Fe@Au/MPD mass ratio and the formation of ordered arrays of Fe@Au nanoparticles. In particular, Fe@Au/MPD $\approx 0.4 \%$ is the optimum mass ratio for forming long-chain arrays of Fe-Au core-shell nanoparticles. Moreover, the results of optical spectroscopy show that the absorbance spectrum of the optimum sample strongly depends on the incoming light polarization, making it a good nominee for optical grating. Finally, a comparison of the Kerr rotations for the optimum sample and the one with an excessive number of cross-linking molecules confirms that the nanoparticles in the latter sample, unlike in the optimum sample, are more densely packed and could not form the ordered arrays.
\end{abstract}

\section{Introduction}

There is a growing interest in the use of Au nanostructures, owing to the local surface plasmon resonances (LSPRs) that manipulate the lightmatter interaction [1,2]. Engineering of the nanoarchitectures for electronics and photonics applications has led to the ordered arrangements of plasmonic nanoparticles [3-6], tailoring the optical properties of the resulting nanoarchitectures by desirably altering the building block constituents and the interparticle distances [3,7-9]. The field enhancement at the interface of a metallic nanoparticle with the underlying dielectric, providing a strong field distribution about the interface, enables the coupling of the plasmonic modes between the adjacent plasmonic components $[5,10]$. Coupling of the plasmonic modes of the adjacent iron-gold core-shell (Fe@Au) nanoparticles arranged in an array has shown to increase the enhancement factor of the surfaceenhanced Raman scattering by two orders of magnitude [11,12]. Moreover, the magnetic properties of the Fe@Au nanoparticles facilitate the manipulation of the inter-particle spacing within an array, via an applied magnetic field [11]. Besides, by an external magnetic field, one can agglomerate the Fe@Au nanoparticles [13]. In other words, these nanoparticles attract each other via their dipolar magnetic fields, forming a one-dimensional supra-structure [14]. Note that since the magnetization saturation of Fe nanoparticles is higher than those of the iron oxides nanoparticles, Fe nanoparticles show a stronger response to a magnetic field [15]. Furthermore, an external applied magnetic field alters the LSPRs electromagnetic field distribution, modifying the optical properties of the nanostructure $[11,12,16,17]$. While the far-field interaction of two adjacent Fe@Au nanoparticles, acting as two magnetic dipoles, is proportional to the inverse of the interparticle spacing, $\mathrm{d}^{-1}$, their near-field plasmonic interaction varies with $\mathrm{d}^{-3}$. Hence, when the interparticle spacing in an array of Fe@Au nanoparticles is much smaller than the incident wavelength $(d \ll \lambda)$, the coupling between adjacent nanoparticles create a linear waveguide for propagation electromagnetic energy below the diffraction limit $[18,19]$. Although noble materials exhibit insignificant magneto-optical phenomena, their integration with magnetic materials or photonic crystal structures can lead to enhanced magneto-optical effects [20-24].

Several methods have been used to fabricate arrays of magnetic and plasmonic nanospheres for developing photonic, electronic, and magnetic devices [5,6,11,17,25-29]. A dilute Langmuir-Blodgett particle monolayer was used to assemble colloidal particles into one-dimensional lines of Au nanoparticles on a substrate [30]. Nonetheless, the colloidal form of the plasmonic nanoparticles can curb their applications in some photonic areas such as integrated optics with a strong desire for film deposition [12,17]. The self-assembling method is another route to fabricate arrays of nanoparticles in a controlled manner

\footnotetext{
* Corresponding author.

E-mail address: moravvej@modares.ac.ir (M.K. Moravvej-Farshi).
} 
$[26,27]$, providing a large scale array of nanoparticles as compared to the ion implantation method [12,17]. Moreover, an engaging magnetic field to assemble arrays of magnetic nanoparticles has been used for the fabrication of an optical grating [31,32]. Others have employed organic molecules, DNA, polymers, and surfactants to align the nanoparticles $[12,22,29,33-36]$. Albeit, there is a need for developing an inexpensive and straight forward method for assembling a large-scale array of Fe@Au nanoparticles with controlled optical properties [37-40].

In this paper, we develop a simple and inexpensive method to assemble a large-scale array of Fe@Au nanoparticles with controlled inter-particle spacing and hence optical properties. In this protocol, we utilize arc discharge that produces no environmental footprints, to prepare high purity iron nanoparticles, followed by growing gold shells around the iron cores with two steps reduction, as compared with the protocols described by $[13,41,42]$. Then, we use m-phenylenediamine (MPD) molecules as conjugated diamines, crosslinking the adjacent synthesized Fe@Au nanoparticles similar to that in $[43,44]$. An ordered array of Fe@Au nanoparticles can be formed by applying an external magnetic field to the substrate, amid the formation of the nanoparticles. Moreover, varying the nanoparticles to MPD molecules mass ratio, we tune the interparticle spacing and hence the optical properties of the arrayed nanoparticles. To the best of our knowledge, this is the first instance of employing MPD as a crosslinker to form arrays of Fe@Au nanoparticles with controlled properties.

\section{Experimental method}

Here, we use the same chemical procedure that we have already described in an earlier report [45]. Fig. 1 (a) shows the synthesizing steps. By creating an electric arc discharge between the two Fe electrodes in deoxygenized deionized water, we synthesize Fe nanoparticles, first. Then, we grow Au seeds, by mixing $20 \mathrm{ml}$ of $0.25 \mathrm{mM}$ solution of gold (III) chloride trihydrate with $5 \mathrm{ml}$ of $0.0049 \mathrm{mM}$ sodium citrate tribasic dihydrate and $0.6 \mathrm{ml}$ of $0.01 \mathrm{M}$ iced sodium borohydride solution. Next, we disperse $0.1 \mathrm{gr}$ of the Fe nanoparticles in $35 \mathrm{ml}$ of toluene and keep the solution in an electric oven at $60{ }^{\circ} \mathrm{C}$ for $4 \mathrm{~h}$, to modify their surface. Then, droplets containing the modified Fe nanoparticles are added to the mixture containing the Au seeds. Next, $20 \mathrm{ml}$ of $1.4 \mathrm{mM}$ solution of gold (III) chloride trihydrate is appended to the product and then $0.4 \mathrm{ml}$ of $78.9 \mathrm{mM}$ acid ascorbic is added to the solution and dispersed in $5 \mathrm{ml}$ ethanol. Finally, we assemble five samples of Fe@Au nanoparticles, each on a glass substrate (see Table 1). As shown in the table, Sample 1 contains no MPD (i.e., the nanoparticles in Sample 1 are not cross-linked), whereas the nanoparticles in Samples 2-5 are cross-linked with different Fe@Au/MPD mass ratios. In fact, each substrate is submerged in a container containing $4.850 \mathrm{ml}$ ethanol and the desired amount of MPD is added (Samples 2-5). Then, we add $150 \mu$ of the solution comprising the synthesized core-sells to each of the five containers, while under an applied magnetic field of $\sim 500 \mathrm{mT}$. At last, the substrates are heated at $70{ }^{\circ} \mathrm{C}$ for $30 \mathrm{~min}$ and dried in air. Fig. 1(b) exemplifies a three-dimensional schematic a substrate on which linear arrays of Fe@Au nanoparticles are formed along an external magnetic field. The inset depicts how two adjacent core-shells are cross-linked via MPD molecules.

Using a Philips EM 208S transmission electron microscope (TEM) at $100 \mathrm{kV}$, we analyze the morphology of the Fe@Au nanoparticles deposited onto the carbon-coated $\mathrm{Cu}$ grid from the dispersed suspension. We also use a Philips diffractometer (model: X'Pert MPD) equipped with a proportional Xe filled detector with radiation of $\mathrm{Cu}-\mathrm{K} \alpha$ at the wavelength of $\lambda=1.54 \AA$ to analyze the X-ray diffraction (XRD) pattern from the nanoparticles. Moreover, using the Fourier-transform infrared spectroscopy (FTIR) Bruker VERTEX $80 \mathrm{~V}$ series, we identify the vibrating and stretching modes of the bonds of the molecules to verify the presence of MPD molecules. Furthermore, employing an Avantespec 3648 UV-visible spectrometer, we assess the electromagnetic interactions between Fe@Au nanoparticles by measuring the variations in the

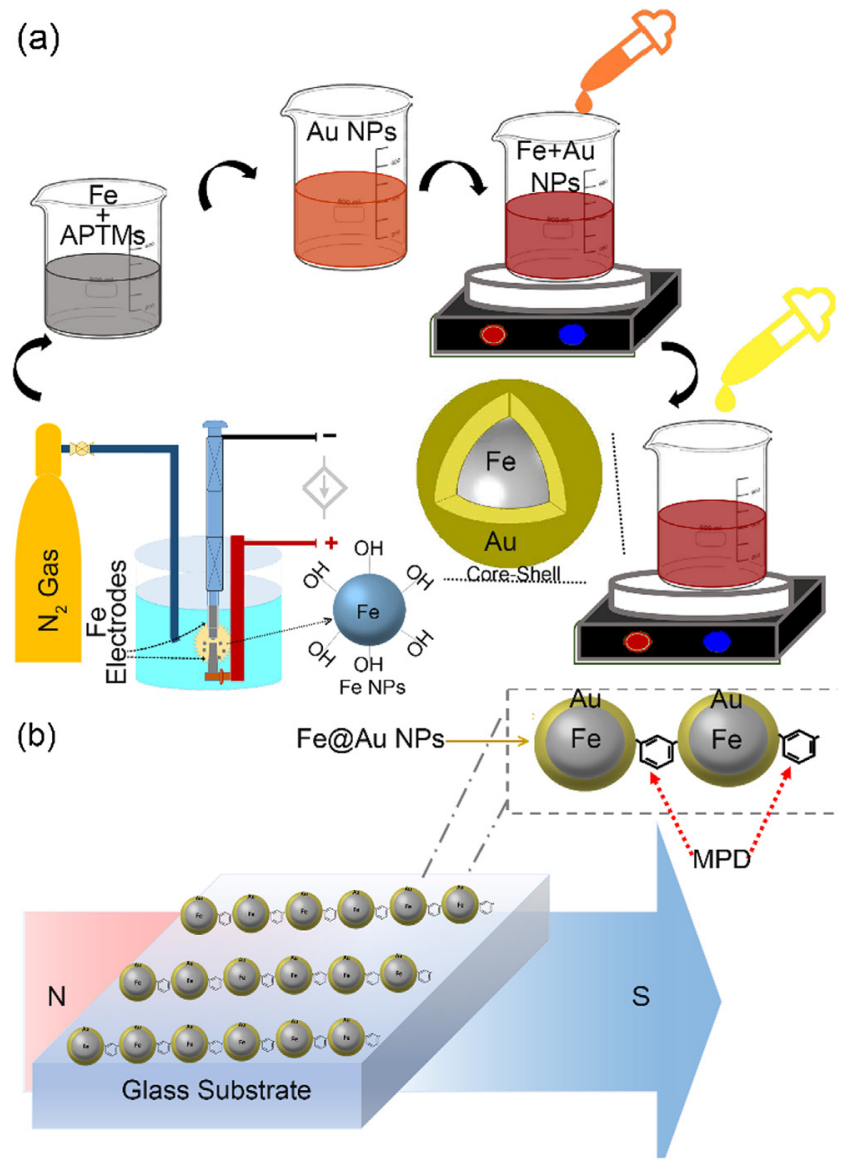

Fig. 1. (a) The schematic of synthesizing Fe@Au nanoparticles; including synthesis of Fe nanoparticles via DC arc discharge, modification nanoparticles surface, and growing Au shells on the modified surfaces, by APTMS, (b) An example of a substrate on which linear arrays of Fe@Au nanoparticles are formed along an external magnetic field. The inset depicts how two adjacent core-shells are cross-linked via MPD molecules.

Table 1

Mass ratios of Fe@Au/MPD in the solution used for preparing Samples 1-5.

\begin{tabular}{llllll}
\hline Sample & 1 & 2 & 3 & 4 & 5 \\
\hline Fe@Au/MPD mass ratio & no MPD & $0.6 \%$ & $0.4 \%$ & $0.12 \%$ & $0.01 \%$ \\
\hline
\end{tabular}

resonance wavelengths of the LSPs as the inter-nanoparticles spacing changes. Moreover, we employ a magneto-optical setup, for both the Kerr effect and Faraday rotation to characterize the magnetic behavior and magneto-optical properties of the Fe@Au nanoparticles. To measure the Kerr rotation and ellipticity simultaneously, we use a $20-\mathrm{mW}$ Helium-Neon laser of wavelength $543.5 \mathrm{~nm}$ modulated by a photoelastic modulator operating at $50 \mathrm{kHz}$ in the setup using Faraday rotation in transmission and Kerr spectrometer in the longitudinal configuration.

\section{Results and discussion}

Fig. 2 characterizes the synthesized Fe@Au nanoparticles. As can be observed in Fig. 2 (a), depicting a TEM image of the as-grown coreshells, each dark sphere that represents a Fe core is covered by a light thin layer that represents the Au shells, forming a spherical Fe@Au nanoparticle. The histogram in Fig. 2(b) illustrates the size distribution of the synthesized Fe@Au nanoparticles that have been estimated from the TEM image, indicating an average particle size of $22 \pm 11 \mathrm{~nm}$. Fig. 2(c) illustrates the optical absorbance spectrum, showing an 


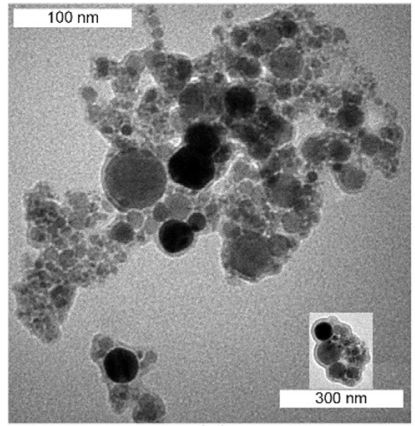

(a)

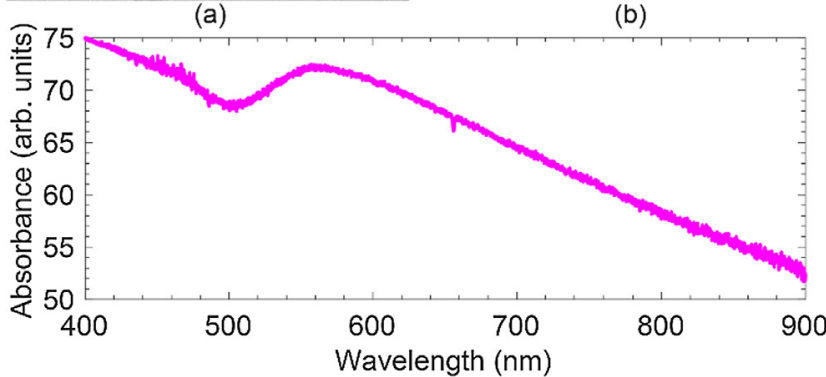

(c)

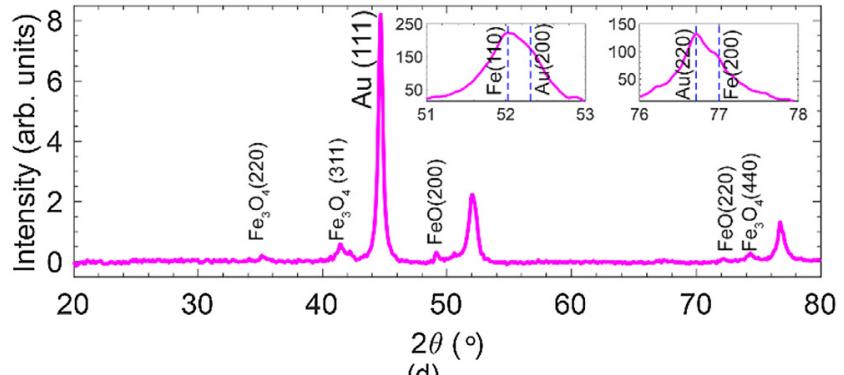

(d)

Fig. 2. (a) TEM image (b) histogram of size distribution that is evaluated from the TEM image, (c) optical absorption spectrum, (d) XRD pattern of the synthesized Fe@Au nanoparticles.

absorption peak at $\lambda=560 \mathrm{~nm}$ that corresponds to the resonance wavelength of the LSPs of the synthesized Fe@Au nanoparticles.

Fig. 2(d) depicts the measured XRD graph, indicating the crystalline planes of iron based materials and Au in the nanoparticles. The peaks in this XRD graph verify the formation Au diffraction planes: $\left(\begin{array}{lll}1 & 1 & 1\end{array}\right)$ at

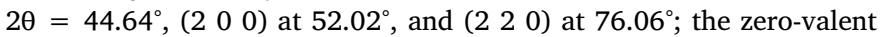
iron crystalline planes: $\left(\begin{array}{lll}1 & 1 & 0\end{array}\right)$ at $51.99^{\circ}$ and $\left(\begin{array}{lll}2 & 0 & 0\end{array}\right)$ at $76.61^{\circ}$; the wustite $\mathrm{Fe}_{\mathrm{x}} \mathrm{O}$ lattice planes ( $\left.\mathrm{x}=0.8-1.0\right):\left(\begin{array}{lll}2 & 0 & 0\end{array}\right)$ at $49.16^{\circ}$ and $\left(\begin{array}{lll}2 & 2 & 0\end{array}\right)$ at $72.06^{\circ}$; and the magnetite $\mathrm{Fe}_{3} \mathrm{O}_{4}$ lattice planes $\left(22^{2} 0\right)$ at $35.11^{\circ}$, (3 11 ) at $41.51^{\circ}$, and (4 40 ) at $74.19^{\circ}$. The zero-valent iron crystalline planes show higher intensity in comparison with wustite and magnetite lattice planes that indicate magnetite and wustite production is negligible in comparison to zero-valent iron.

To verify the interaction between MPD molecules and nanoparticles, we compare the FTIR spectra of two samples similar to Samples 1 and 3 that are deposited on ZnS substrates rather than glass substrates, because of the transparency of $\mathrm{ZnS}$ to the IR wavelengths. Fig. 3 compares the measured absorbance spectra of both samples with similar sputum obtain from a bare ZnS substrate. The dots represent the spectrum from Sample 1 (no MPD), the dashes represent the spectrum from Sample 3, and the solid curve depicts the spectrum from the bare $\mathrm{ZnS}$ substrate. The Characteristic peaks located at the wavenumber $567 \mathrm{~cm}^{-1}$, appearing on the spectra of Samples 1 and 3, are assigned to the stretching modes of Fe-O bonds at the tetrahedral sites [46]. The spectrum of the ZnS substrate does not exhibit this characteristic. The peaks that solely appear on the spectra of Sample 3 at the wavenumbers 3320 and $3212 \mathrm{~cm}^{-1}$ are attributed to the N-H bonds stretches, as a consequence

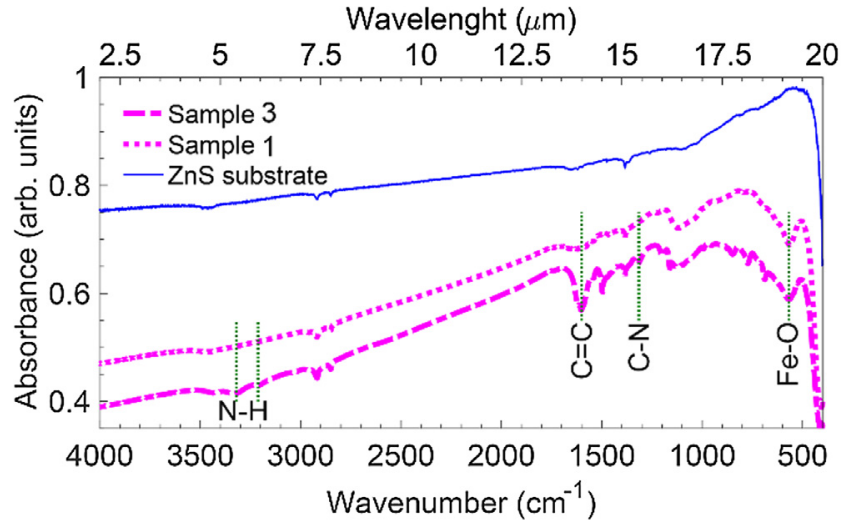

Fig. 3. FTIR spectra of Fe@Au nanoparticles similar to those of Sample 1 (dots) and Sample 3 (dots-dashes) formed on ZnS substrates are compared with the absorbance spectrum of a bare ZnS substrate.

of introducing MPD molecule [43]. Moreover, the peak also observed on the spectra of Sample 3 at wavenumbers 1600 and $1315 \mathrm{~cm}^{-1}$ are ascribed to the $\mathrm{C}=\mathrm{C}$ and $\mathrm{C}-\mathrm{N}$ stretches, due to the presence of MPD molecules in the sample [43].

Fig. 4 demonstrates the optical microscopic images of the five samples of Table 1. Due to the nanoparticles' magnetic properties, they are arrayed along the magnetic field. Comparing these five images shows that the image in Fig. 4(b) is more or less like that of Fig. 4(a). In other words, the number of MPD molecules in Samples 2 was not sufficient enough to produce arrays of core-shells. Nonetheless, the image in Fig. 4(c) indicates that Sample 3 contained a sufficient number of MPD molecules to produce periodic arrays of Fe@Au nanoparticles. This is because the van der Waals interaction in competition with the dipole-dipole interaction has succeeded in creating large-scale orderly arrays interlinked nanoparticles. However, an excessive number of MPD molecules in Samples 4 and 5 have resulted in the aggregated clusters of Fe@Au nanoparticles (Fig. 4(d) and (e)).

The interactions between two adjacent Fe@Au nanoparticles are mainly subject to the distance between the two particles and their radii. Nonetheless, the smaller the nanoparticle the more important the van der Waals interaction [47].

Fig. 5 compares the FE-SEM images of Samples 1-5. This comparison also reveals the critical role of the number of MPD molecules in the arrangement of the nanoparticles arrays, confirming the results observed in the optical images of the corresponding samples. The FE-SEM image in Fig. 5(c) confirms that a sufficient number of MPD molecules have interlinked each Fe@Au with its neighboring nanoparticles, arranging them in arrays along the direction of the applied external magnetic field during the fabrication process, in Sample 3.

Next, we compare the optical absorbance spectra obtained for Samples 1-5 with a similar spectrum from a solution containing the synthesized Fe@Au nanoparticles, to observe the effect of the amount of MPD molecules on the optical features of the samples. Fig. 6 illustrates this comparison. The thin blue dashes represent the absorbance spectrum for the Fe@Au nanoparticles suspended in the solution before the addition of MPD. The thin pink solid line represents that of Sample 1 (i.e. with no MPD) and the thick pink dots-dashes, solid line, dashes, and dots represent the spectra for Samples 2-5 and. Each spectrum is an indicator of the arrangement of nanoparticles formed in the corresponding environment.

The inset illustrates a zoomed-in view of the plots, comparing the plasmonics absorbance spectra around their peaks. This observation, confirming our discussions about Fig. 4(a) and Fig. 5(a), indicate that Sample 1 containing no crosslinkers constitutes ensembles of closely packed disordered Fe@Au nanoparticles. On the other hand, extremely small ratio of Fe@Au/MPD (i.e., 0.01\%) in Sample 5 has aggregated the arrayed nanoparticles into large clusters of $\mathrm{Fe} @ \mathrm{Au}$ nanoparticles, 


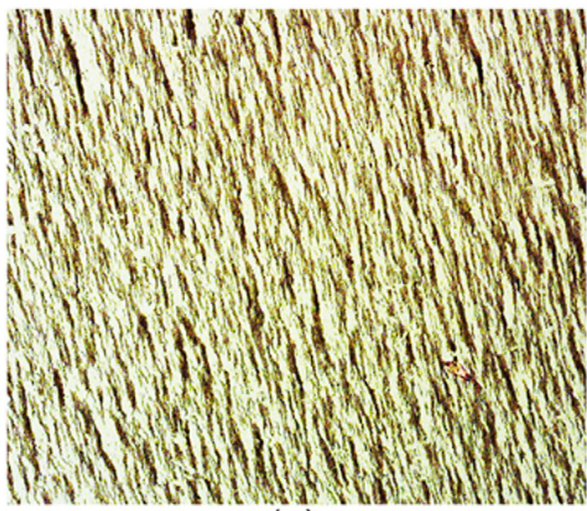

(a)

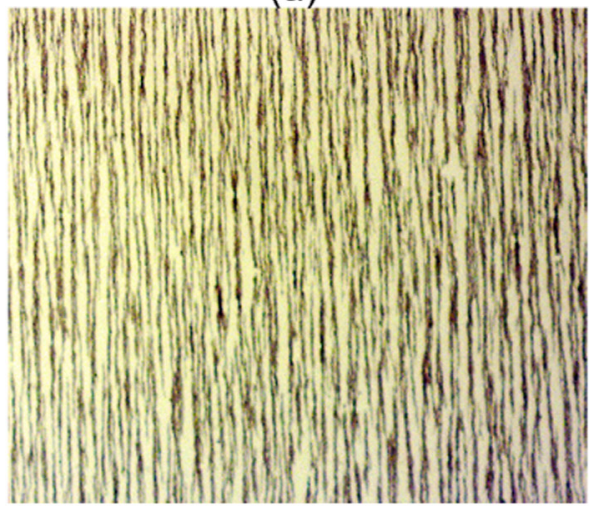

(c)

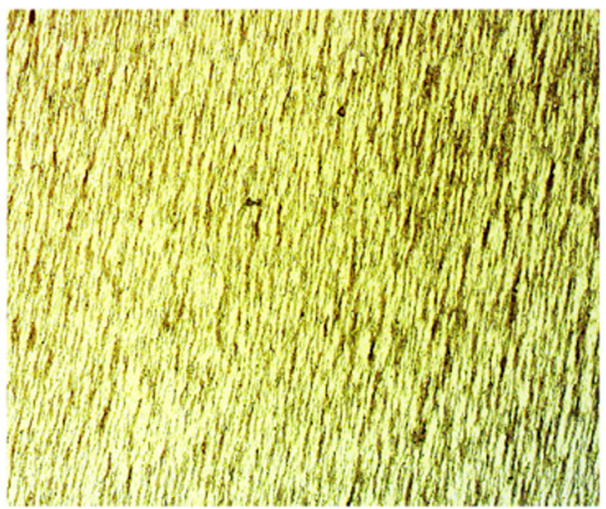

(b)

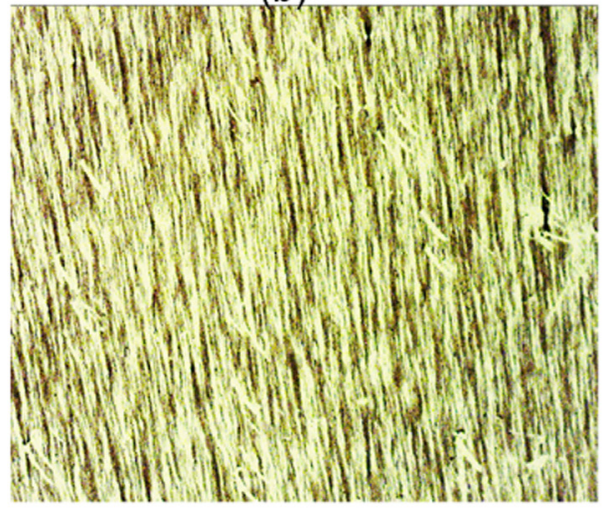

(d)

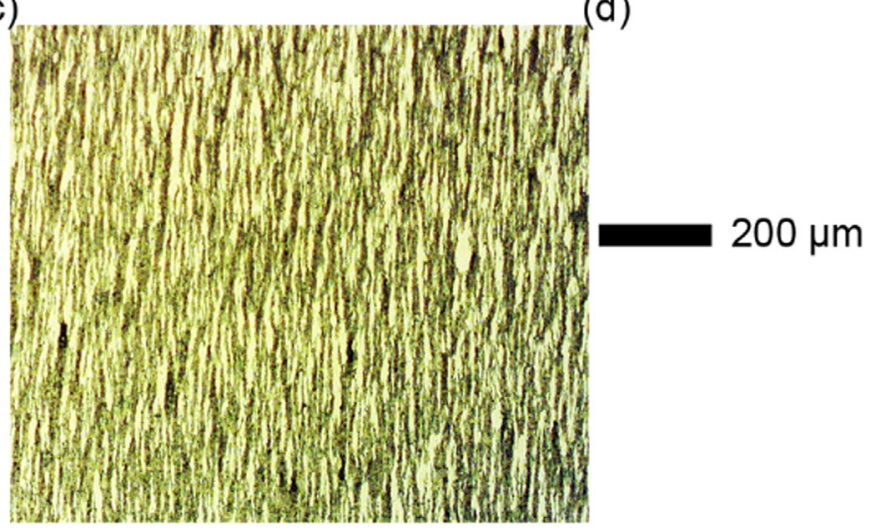

(e)

Fig. 4. Optical microscopic images of nanoparticles assembled on the glass substrates of (a) Sample 1, (b) Sample 2, (c) Sample 3, and (d) Sample 4, (e) Sample 5, under the same external magnetic field.

resulting in the broadest spectrum depicted by the thick dots, confirming the discussion about corresponding optical and FE-SEM images (Fig. 4(e) and Fig. 5(e)). Among the three Samples 2-4, the spectra for Samples 2 and 4 are broader than that of Sample 3, confirming the comparisons regarding the corresponding images in Fig. 4 and Fig. 5. In other words, Sample 2 with the largest absorbance peak, containing an insufficient number of crosslinkers is composed of some arrayed Fe@Au nanoparticles mixed with dispersed nanoparticles between them, whilst Sample 3 with a sufficient number of crosslinkers contains ordered arrays of $\mathrm{Fe}-\mathrm{Au}$ nanoparticles. Moreover, an excessive number of crosslinkers in Samples 4 resulted in some aggregated clusters of Fe@Au nanoparticles, exhibiting an absorbance spectrum that is the broadest amongst the three samples. These observations reveal that there is a tradeoff between the Fe@Au/MPD mass ratio and the formation of ordered arrays of Fe@Au nanoparticles.

Next, we investigate the effect of the incident light polarization on the plasmonic absorption in Sample 3. For the sake of comparison, we revisit the plasmonic absorption spectra in Sample 3 for unpolarized incident light, as depicted by the blue dashes in Fig. 7. Using a P-polarized incident light, we repeated the measurement once when the polarization vector is parallel with the chain interlinking the nanoparticles in the array (red solid line). The inset shows that the peak of this spectrum is suppressed $\sim 8.8 \%$ redshifted by $\sim 12 \mathrm{~nm}$ with respect to that of the unpolarized light.

Then, rotating the sample by $90^{\circ}$, we repeated the measurement once again (pink dots). The inset shows that the peak of the latter spectrum while experiencing a larger suppression $(\sim 13 \%)$ exhibits a smaller redshift $(\sim 7 \mathrm{~nm})$ with respect to that of the unpolarized light. These observations reveal that the effective dielectric constant of the arrayed nanoparticles in response to the P-polarized light depends on the orientation of the nanoparticles chain with respect to the polarization vector, showing a stronger interparticle interaction (near-field coupling between the adjacent nanoparticles) when the polarization vector is parallel to the array chain created by the MPD molecules. 


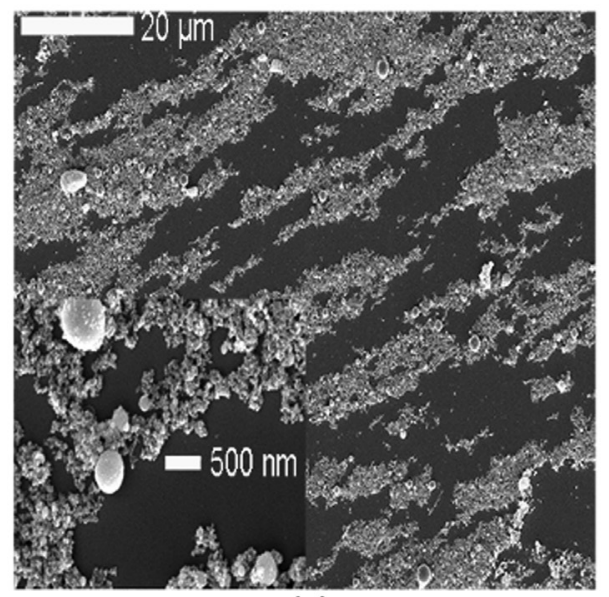

(a)

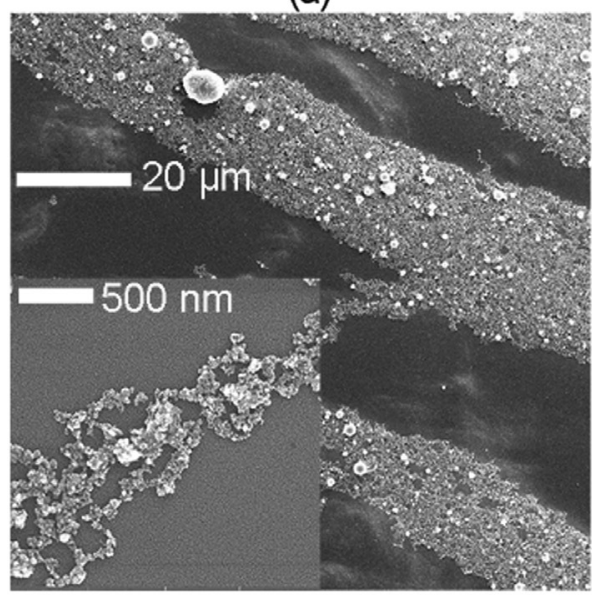

(c)

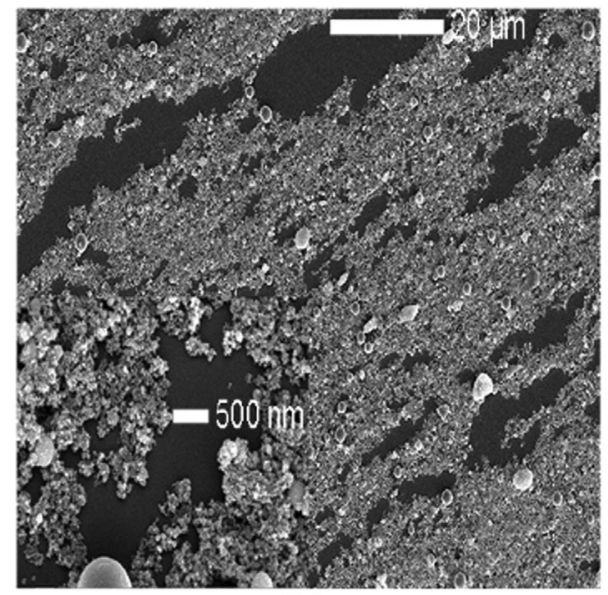

(b)

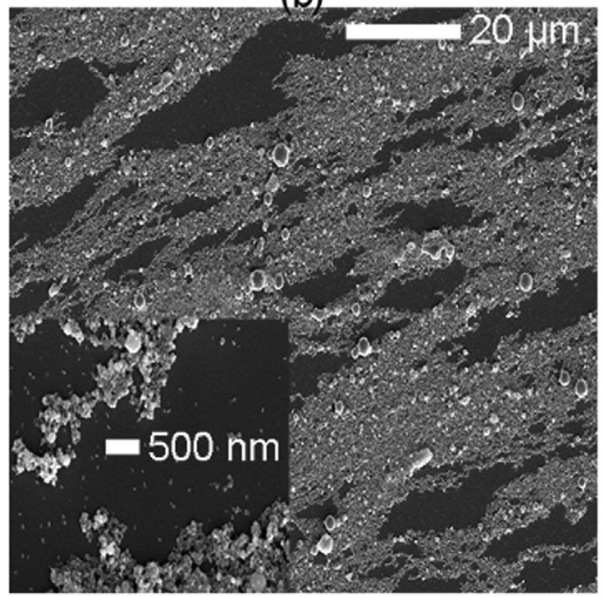

(d)

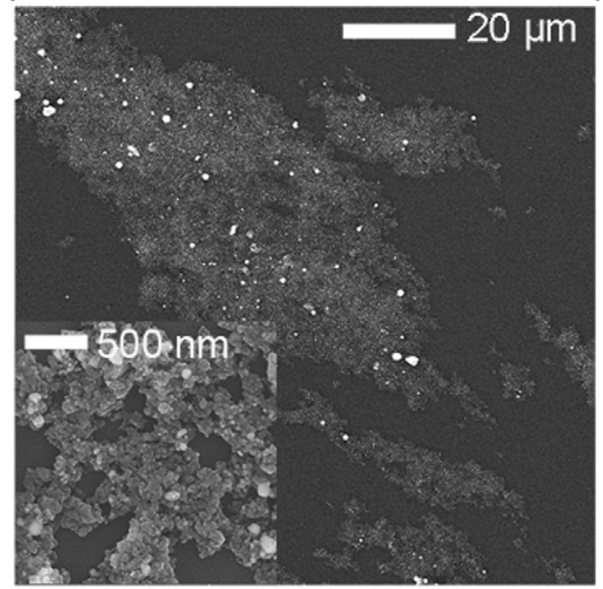

(e)

Fig. 5. FE- SEM image of the assembly of (a) Sample 1, (b) Sample 2, (c) Sample 3, (d) Sample 4, (e) and Sample 5.

Nonetheless, the blue dashes reveal that the interparticle interaction for the unpolarized light is stronger than those in the presence of the ppolarized lights (whether for @ $0^{\circ}$ or @ $90^{\circ}$ ). These experimental results behave similarly to those reported by others $[7,8,18]$.

Finally, we have measured the magneto-optical effects, including the Kerr rotation, ellipticity and the Faraday rotation in reflection and transmission light, for Sample 3 and Sample 5. The solid circles in Fig. 8(a) represent the Kerr rotation for Sample 3, while the open circles depict the same characteristic for Sample 5. Similar legends in Fig. 8(b) represent the corresponding ellipticity for Samples 3 and 5. Fig. 8(c) depicts the data for the Faraday rotation obtained for Samples 3 (solid circle) and 5 (open circles). Comparing the latter sets of data confirms that the nanoparticles in Sample 5 due to the excessive number of crosslinking molecules, unlike in Sample 3, could not form an ordered array. In other words, the nanoparticles in Sample 5 are more densely packed than those are in Sample 3. These measured data are in good agreement with those reported by [37]. The Faraday and Kerr rotations, both display the same magnetic activity. The iron cores in Fe@Au nanoparticles behave superparamagnetically and do not exhibit a significant hysteresis. Moreover, the Faraday rotation in both samples denotes the effect of magnetization of iron cores and is unable to demonstrate the contribution of the plasmonic effect. 


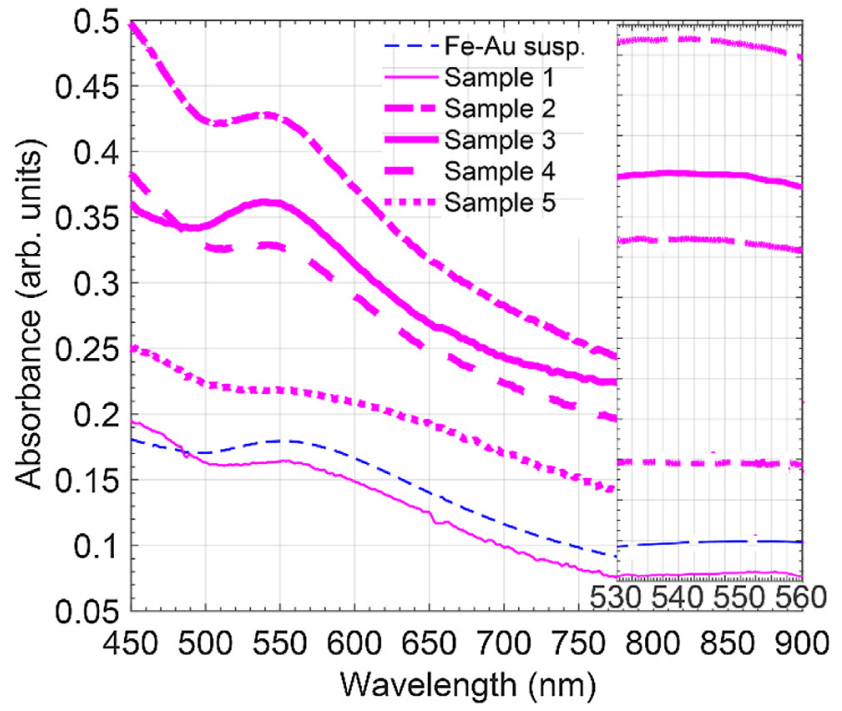

Fig. 6. Comparison of the optical absorbance spectra obtained from the Fe@Au nanoparticles in solution (thin blue dashes) and those of Samples 1 (thin solid line), 2 (thick solid line), 3(thick dots-dashes) 4, (thick dashes), and 5 (thick dots).

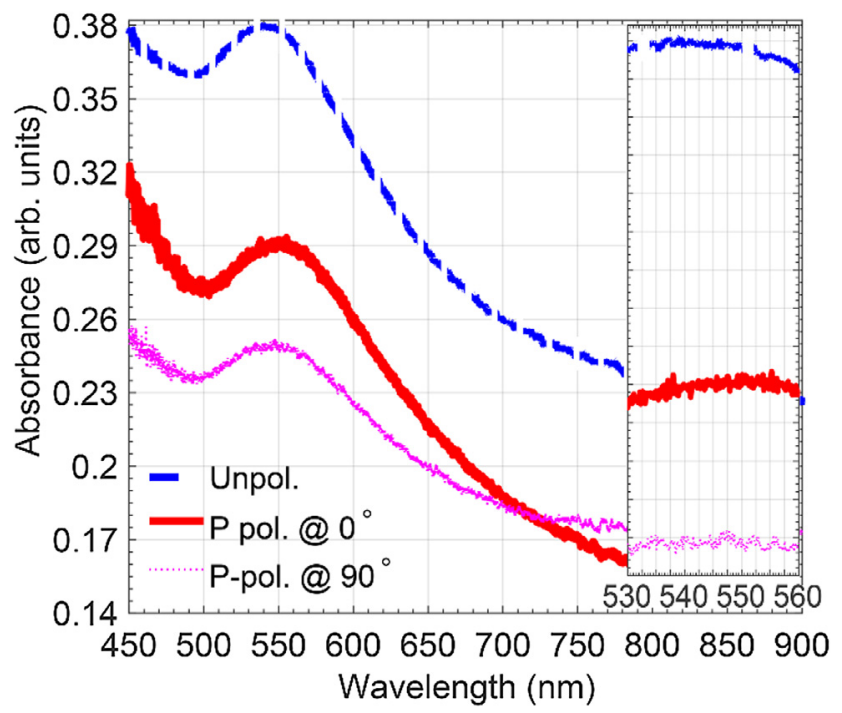

Fig. 7. Comparison of the optical absorbance spectra from the arrays of Fe@Au nanoparticles formed on Sample 3, experimentally obtained when the incident optical signal is unpolarized (blue dashes), p-polarized (@0 $0^{\circ}$ : red solid line) and (@90: pink dots).

\section{Conclusion}

We have presented an innovative low cost process to form the arrays of the Fe@Au nanoparticles. To form ordered arrays of nanoparticles, we synthesized MPD molecules as a crosslinker between nanoparticles under the magnetic field. The optical absorbance spectroscopy has revealed that as the Fe@Au/MPD mass ratio increases, the corresponding plasmon resonance becomes intensified with respect to that of the sample with no MPD molecules. Nevertheless, images of optical microscopy and FE-SEM have verified the existence of a tradeoff between the Fe@Au/MPD mass ratio and the formation of long-chain arrays of crosslinked Fe@Au nanoparticles, showing Fe@Au/MPD $\approx 0.4 \%$ is the nearly optimum mass ratio. Moreover, the optical absorbance spectroscopy has shown that the resulting spectrum for the optimum sample depends on the incoming light polarization, revealing the near-field coupling between the adjacent nanoparticles in response to an

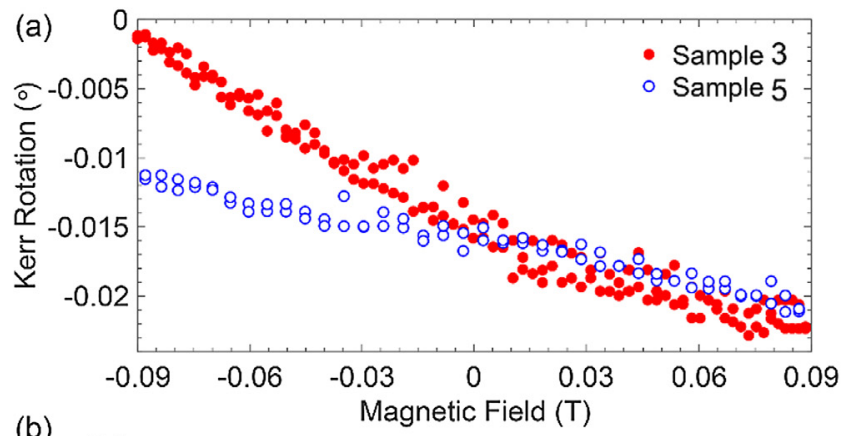

(b)
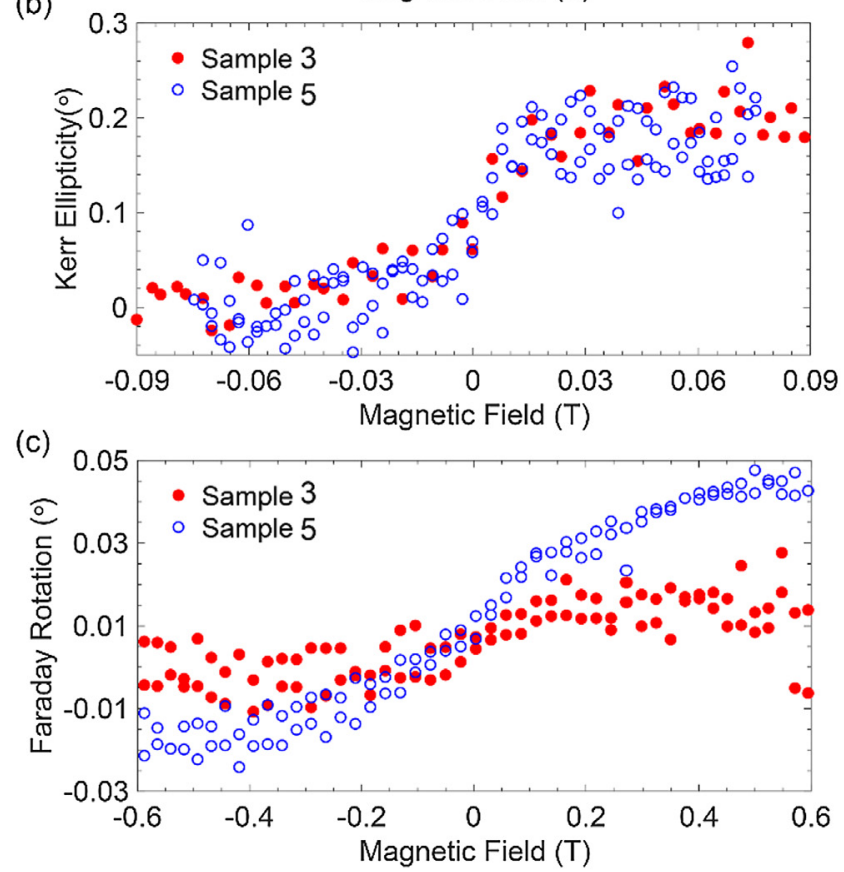

Fig. 8. The measured (a) Kerr rotation, (b) Kerr ellipticity, (c) Faraday rotation for arrays of Fe@Au nanoparticles formed in Sample 3 (solid circles) and Sample 5 (open circles).

unpolarized light is stronger than those in response to the p-polarised light with polarization vectors parallel or perpendicular to the direction of the arrayed chains. These plasmonic coupling behavior make the optimum sample a good nominee as optical grating.

\section{CRediT authorship contribution statement}

Najme Ahmadi: Conceptualization, Data curation, Formal analysis, Investigation, Methodology, Writing the original draft. Reza Poursalehi: Supervision, Validation, Visualization, Writing - review \& editing. Andrei Kirilyuk: Validation, Writing - review \& editing. Mohammad Kazem Moravvej-Farshi: Funding acquisition, Project administration, Resources, Supervision, Writing - review \& editing.

Declaration of Competing Interest

The authors declare that they have no known competing financial interests or personal relationships that could have appeared to influence the work reported in this paper.

\section{Acknowledgments}

N. Ahmadi, R. Poursalehi and M. K. Moravvej-Farshi gratefully acknowledge the financial support received from Tarbiat Modares University, through grant \#IG-39703.

N. Ahmadi would also like to thank Dr. F. Arabpour-Roghabadi for her kindly discussion regarding the chemical processes and K. Saeed 
Ilkhchy for his assistance in FTIR measurements.

\section{References}

[1] J.Y. Chin, T. Steinle, T. Wehlus, D. Dregely, T. Weiss, V.I. Belotelov, B. Stritzker, H. Giessen, Nonreciprocal plasmonics enables giant enhancement of thin-film Faraday rotation, Nat. Commun. 4 (2013) 1599.

[2] A. Hohenau, J.R. Krenn, J. Beermann, S.I. Bozhevolnyi, S.G. Rodrigo, L. MartinMoreno, F. Garcia-Vidal, Spectroscopy and nonlinear microscopy of Au nanoparticle arrays: Experiment and theory, Phys. Rev. B 73 (2006) 155404.

[3] S.J. Tan, M.J. Campolongo, D. Luo, W. Cheng, Building plasmonic nanostructures with DNA, Nat. Nanotechnol. 6 (2011) 268.

[4] B. Lamprecht, G. Schider, R. Lechner, H. Ditlbacher, J.R. Krenn, A. Leitner, F.R. Aussenegg, Metal nanoparticle gratings: influence of dipolar particle interaction on the plasmon resonance, Phys. Rev. Lett. 84 (2000) 4721.

[5] S.K. Ghosh, T. Pal, Interparticle coupling effect on the surface plasmon resonance of gold nanoparticles: from theory to applications, Chem. Rev. 107 (2007) 4797-4862.

[6] T. Kim, K. Lee, M.-S. Gong, S.-W. Joo, Control of gold nanoparticle aggregates by manipulation of interparticle interaction, Langmuir 21 (2005) 9524-9528.

[7] W. Rechberger, A. Hohenau, A. Leitner, J. Krenn, B. Lamprecht, F. Aussenegg, Optical properties of two interacting gold nanoparticles, Opt. Commun. 220 (2003) 137-141.

[8] M. Quinten, A. Leitner, J.R. Krenn, F.R. Aussenegg, Electromagnetic energy transport via linear chains of silver nanoparticles, Opt. Lett. 23 (1998) 1331-1333.

[9] S. Kinge, M. Crego-Calama, D.N. Reinhoudt, Self-assembling nanoparticles at surfaces and interfaces, ChemPhysChem 9 (2008) 20-42.

[10] M. Caminale, L. Anghinolfi, E. Magnano, F. Bondino, M. Canepa, L. Mattera, F. Bisio, Tuning the magneto-optical response of iron oxide nanocrystals in Au-and Ag-based plasmonic media, ACS Appl. Mater. Inter. 5 (2013) 1955-1960.

[11] F. Hu, H. Lin, Z. Zhang, F. Liao, M. Shao, Y. Lifshitz, S.-T. Lee, Smart liquid SERS substrates based on $\mathrm{Fe} 3 \mathrm{O}$ 4/Au nanoparticles with reversibly tunable enhancement factor for practical quantitative detection, Sci. Rep. 4 (2014) 7204.

[12] L. Polavarapu, Q.-H. Xu, Water-soluble conjugated polymer-induced self-assembly of gold nanoparticles and its application to SERS, Langmuir 24 (2008) 10608-10611.

[13] W. Zhou, E. Carpenter, J. Lin, A. Kumbhar, J. Sims, C. O'Connor, Nanostructures of gold coated iron core-shell nanoparticles and the nanobands assembled under magnetic field, Eur. Phys. J. D. 16 (2001) 289-292.

[14] A. Weddemann, F. Wittbracht, B. Eickenberg, A. Hütten, Magnetic field induced assembly of highly ordered two-dimensional particle arrays, Langmuir 26 (2010) 19225-19229.

[15] J.M.D. Coey, J.M.D. Coey, Magnetism and Magnetic Materials, Cambridge University Press, 2010.

[16] T. Ding, K. Song, K. Clays, C.H. Tung, Fabrication of 3D photonic crystals of ellipsoids: convective self-assembly in magnetic field, Adv. Mater. 21 (2009) 1936-1940.

[17] X. Xue, J. Wang, E.P. Furlani, Self-assembly of crystalline structures of magnetic core-shell nanoparticles for fabrication of nanostructured materials, ACS Appl. Mater. Inter. 7 (2015) 22515-22524.

[18] S.A. Maier, M.L. Brongersma, P.G. Kik, H.A. Atwater, Observation of near-field coupling in metal nanoparticle chains using far-field polarization spectroscopy, Phys. Rev. B 65 (2002) 193408.

[19] S.A. Maier, P.G. Kik, H.A. Atwater, S. Meltzer, E. Harel, B.E. Koel, A.A. Requicha, Local detection of electromagnetic energy transport below the diffraction limit in metal nanoparticle plasmon waveguides, Nat. Mater. 2 (2003) 229.

[20] D. Regatos, D. Fariña, A. Calle, A. Cebollada, B. Sepúlveda, G. Armelles, L.M. Lechuga, Au/Fe/Au multilayer transducers for magneto-optic surface plasmon resonance sensing, J. Appl. Phys. 108 (2010) 054502.

[21] M. Afsharnia, S.M. Hamidi, Characterization of $\mathrm{Au} / \mathrm{Fe} / \mathrm{Au}$ and $\mathrm{Au} / \mathrm{Co} / \mathrm{Au}$ MagnetoPlasmonic Multilayers as an Ethanol Vapor Sensor, IEEE Trans. Magn. 54 (2018) 1-7.

[22] C. de Julián Fernández, G. Mattei, E. Paz, R. Novak, L. Cavigli, L. Bogani, F. Palomares, P. Mazzoldi, A. Caneschi, Coupling between magnetic and optical properties of stable Au-Fe solid solution nanoparticles, Nanotechnology 21 (2010) 165701.

[23] G. Wurtz, W. Hendren, R. Pollard, R. Atkinson, L. Le Guyader, A. Kirilyuk,
T. Rasing, I. Smolyaninov, A. Zayats, Controlling optical transmission through magneto-plasmonic crystals with an external magnetic field, New J. Phys. 10 (2008) 105012.

[24] P.K. Jain, Y. Xiao, R. Walsworth, A.E. Cohen, Surface plasmon resonance enhanced magneto-optics (SuPREMO): Faraday rotation enhancement in gold-coated iron oxide nanocrystals, Nano Letters 9 (2009) 1644-1650.

[25] X. Xue, K. Liu, E.P. Furlani, Theoretical study of the self-assembly and optical properties of 1D chains of magnetic-plasmonic nanoparticles, J. Phys. Chem. C. 121 (2017) 9489-9496.

[26] S. Bai, Q. Li, H. Zhang, X. Chen, S. Luo, H. Gong, Y. Yang, D. Zhao, M. Qiu, Large third-order nonlinear refractive index coefficient based on gold nanoparticle aggregate films, Appl. Phy. Lett. 107 (2015) 141111.

[27] J. Ge, Y. Yin, Magnetically tunable colloidal photonic structures in alkanol solutions, Adv. Mater. 20 (2008) 3485-3491.

[28] J. Ge, Y. Hu, Y. Yin, Highly tunable superparamagnetic colloidal photonic crystals, Angew. Chem. 119 (2007) 7572-7575.

[29] H. Hu, H. Zhong, C. Chen, Q. Chen, Magnetically responsive photonic watermarks on banknotes, J. Mater. Chem. C 2 (2014) 3695-3702.

[30] J. Huang, A.R. Tao, S. Connor, R. He, P. Yang, A general method for assembling single colloidal particle lines, Nano Letters 6 (2006) 524-529.

[31] J. Henderson, S. Shi, S. Cakmaktepe, T. Crawford, Pattern transfer nanomanufacturing using magnetic recording for programmed nanoparticle assembly, Nanotechnology 23 (2012) 185304.

[32] L. Ye, B. Terry, O. Mefford, C. Rinaldi, T. Crawford, All-nanoparticle concave diffraction grating fabricated by self-assembly onto magnetically-recorded templates, Opt. express 21 (2013) 1066-1075.

[33] J. Sharma, R. Chhabra, Y. Liu, Y. Ke, H. Yan, DNA-templated self-assembly of twodimensional and periodical gold nanoparticle arrays, Angew. Chem. 118 (2006) 744-749.

[34] Q. Ma, E.E. Remsen, T. Kowalewski, K.L. Wooley, Two-dimensional, shell-crosslinked nanoparticle arrays, J. Am. Chem. Soc. 123 (2001) 4627-4628.

[35] Y. Yang, L. Xiong, J. Shi, M. Nogami, Aligned silver nanorod arrays for surfaceenhanced Raman scattering, Nanotechnology 17 (2006) 2670.

[36] Y.J. Li, W.J. Huang, S.G. Sun, A universal approach for the self-assembly of hydrophilic nanoparticles into ordered monolayer films at a toluene/water interface, Angew. Chem. 45 (2006) 2537-2539.

[37] T. Fried, G. Shemer, G. Markovich, Ordered two-dimensional arrays of ferrite nanoparticles, Adv. Mater. 13 (2001) 1158-1161.

[38] J. Yuan, Y. Xu, A.H. Mueller, One-dimensional magnetic inorganic-organic hybrid nanomaterials, Chem. Soc. Rev. 40 (2011) 640-655.

[39] T. Chen, M. Pourmand, A. Feizpour, B. Cushman, B.R.M. Reinhard, Tailoring plasmon coupling in self-assembled one-dimensional Au nanoparticle chains through simultaneous control of size and gap separation, J. Phys. Chem. Lett. 4 (2013) 2147-2152.

[40] M.L. Brongersma, N.J. Halas, P. Nordlander, Plasmon-induced hot carrier science and technology, Nat. Nanotechnol. 10 (2015) 25.

[41] S.H. Khezri, A. Yazdani, R. Khordad, Effect of characteristics of media on cobalt and iron nanoparticles prepared by arc discharge method, J. Ind. Eng. Chem. 20 (2014) 521-527.

[42] L. Li, M. Fan, R.C. Brown, J. Van Leeuwen, J. Wang, W. Wang, Y. Song, P. Zhang, Synthesis, properties, and environmental applications of nanoscale iron-based materials: a review, Crit. Rev. Environ. Sci. Technol. 36 (2006) 405-431.

[43] F.A. Roghabadi, M. Kokabi, V. Ahmadi, G. Abaeiani, Quantum dots crosslinking as a new method for improving charge transport of polymer/quantum dots hybrid solar cells and fabricating solvent-resistant film, Electrochim. Acta 222 (2016) 881-887.

[44] J.J. Martínez, H. Rojas, L. Vargas, C. Parra, M.H. Brijaldo, F.B. Passos, Hydrogenation of m-dinitrobenzene over Au catalysts on magnetic supports, J. Mol. Catal. A. 383 (2014) 31-37.

[45] N. Ahmadi, R. Poursalehi, A. Kirilyuk, M.K. Moravvej-Farshi, Effect of gold plasmonic shell on nonlinear optical characteristics and structure of iron based nanoparticles, Appl. Surf. Sci. 479 (2019) 114-118.

[46] A. Sood, V. Arora, J. Shah, R. Kotnala, T.K. Jain, Multifunctional gold coated iron oxide core-shell nanoparticles stabilized using thiolated sodium alginate for biomedical applications, Mat. Sci. Eng. C 80 (2017) 274-281.

[47] G.N. Rao, Y. Yao, Y. Chen, K. Wu, J. Chen, Particle size and magnetic field-induced optical properties of magnetic fluid nanoparticles, Phys. Rev. E 72 (2005) 031408. 